\title{
CLINICAL TRIALS IN DEVELOPING COUNTRIES - ETHICAL CONSIDERATIONS
}

\author{
Marija Bosnjak Pasic ${ }^{1,2}$, Branka Vidrih ${ }^{3}$, Helena Sarac ${ }^{2}$, Hanna Pasic ${ }^{4}$, Luka Vujević ${ }^{4}$, \\ Anamarija Soldo Koruga ${ }^{5}$ \& Fabijan Rajič ${ }^{6}$ \\ ${ }^{1}$ School of Medicine, University of Osijek, Osijek, Croatia \\ ${ }^{2}$ Department of Neurology, University Hospital Centre Zagreb, School of Medicine, \\ University of Zagreb,Zagreb, Croatia \\ ${ }^{3}$ Department of Psychiatry, University Hospital Centre Sisters of Mercy, \\ Catholic University of Croatia, Zagreb, Croatia \\ ${ }^{4}$ student, School of Medicine, University of Zagreb, Zagreb, Croatia \\ ${ }^{5}$ Department od Neurology, University Hospital Centar Osijek, Department of Neurology and Neurosurgery, \\ Faculty of Medicine, Josip Juraj Strossmayer University of Osijek, Osijek, Croatia \\ ${ }^{6}$ Croatian Football Federation, Zagreb Football Association, Zagreb, Croatia
}

received: 23.3.2018;

revised: 7.6.2018;

accepted: 3.7 .2018

\section{SUMMARY}

When designing clinical trial or considering decision to take part in particular clinical trial as investigators, even before submission to responsible Central Ethic Committee, we always make certain private assessment about ethical justification of this clinical trial. When making assessment if any clinical trial is ethically justifiable, there should make no difference in which country this clinical trial will be executed. Physicians coming from developing countries must ensure that patient population of developing countries is not misused in any ethically questionable clinical trial. There must be careful assessment of clinical protocols by various independent local advisory committees (e.g. hospital review boards, hospital drug committees, hospital administration and whatever is applicable) to exclude the possibility that only one person or one group of people has concentrated power to make decisions for entire country. Many times physicians/clinical researchers from developing countries are faced with the criticisms that they are not of the same quality as physicians from developed countries and that they can be easily bribed by sponsors, which are based on the prejudice that any clinical trial can be executed in developing countries, no matter of quality or risks for patients. Physicians coming from developing countries must ensure that patient population of developing countries is not misused in any ethically questionable clinical trial.

Key words: clinical trials - developing countries - ethics

\section{INTRODUCTION}

When designing clinical trial or considering decision to take part in particular clinical trial as investigators, even before submission to responsible Central Ethic Committee, we always make certain private assessment about ethical justification of this clinical trial. When making assessment if any clinical trial is ethically justifiable, there should make no difference in which country this clinical trial will be executed. There should be no difference how scientific and medical rationale will be justified, and there should not be different standards for best medical care if the study patients would come from developing countries, which are grouped as such by The World Bank following GDP per capita standard - taken as a parameter of development that predominantly consists of countries from Africa, Asia or South America, and also European countries such as Srbia, Bosnia and Herzegovina, Kosovo, Albania, Bulgaria etc. Standard of care may not be the same for all patients in all the countries where clinical trial will be performed, but even in developing countries there are a number of patients who can afford and are receiving the best standard of medical care. If the standard of care provided in clinical trial would not be the same for all patients including those coming from developing countries, this will increase probability that patients would come from vulnerable population and do not have many choices, but are ready to accept lower standards. In such a way risk/benefit evaluation will be different as more risks would be accepted, clinical equipoise will not be reached and scientific value of clinical trial would be decreased. Here also comes the question what is the fair remuneration for Investigators participating in clinical trials coming from developing countries. Many times their fee for participation in clinical trial is somewhat lower than for physicians coming from developed countries. The rule of fair market value, applied by the sponsors of the clinical trial is not very clear rule. Many times physicians/clinical researchers from developing countries are faced with the criticisms that they are not of the same quality as physicians from developed countries and that they can be easily bribed by sponsors, which are based on the prejudice that any clinical trial can be executed in developing countries, no matter of quality or risks for patients. Physicians 
coming from developing countries must ensure that patient population of developing countries is not misused in any ethically questionable clinical trial. There must be a careful assessment of clinical protocols by various independent local advisory committees (e.g. hospital review boards, hospital drug committees, hospital administration and whatever is applicable) to exclude the possibility that only one person or one group of people has concentrated power to make decisions for entire country. Nuffield Report recommends that all developing countries should have in place a properly constituted and functioning system for the independent ethical review of research. This will include establishing effective control boards with function of researching ethical sciences and better surveillance through Ethical comittees that will supervise and assess ethical justification of clinical research and methods of the trial itself, and will also answer about their procedures and finds to the overarching institution responsible for the region of countries in which it is established, and such are

European Medicines Agency (EMA), Food and Drug Administration (FDA), etc. In that way pharmaceutical industry and entire scientific branch that performs clinical researches will shift their focus towards new, more ethical as well as humane concept of testing drugs, seeking for mutual close relations between all of the fields and institutions in specific clinical trials until the new pharmaceutic reaches its market (Verbanac et al. 2013; EMA). If high standards of ethical research would not be followed, physician's credibility among patients will be lost. There is always possibility to reject ethically questionable clinical trials and flawed ideas. Sometimes it requires personal courage, sometimes it is connected with financial or career losses, but all this is only temporary hardship. What remains is physician's integrity and sincere wish to help patients by clinical, scientific research.

Every clinical trial, no matter if it will be executed in developed or developing countries in order to be considered as ethical clinical research, must adhere to the seven key requirements of ethical research (Emanuel et al. 2000). The aim of this paper is to discuss ethical requirements of ethical research and to present critical aspects of clinical trials.

\section{RECRUITMENT TO CLINICAL TRIALS - CRITICAL ASPECTS}

When offering participation in clinical trial, physician must make every effort to explain to the patient which other options for the treatment exist, not just in the country where the patient lives, but also worldwide, as we never know what other options patient may have to get this treatment (e.g. family abroad, fund raising etc.). The decision about participation in clinical trial should not come out as the only option for the patient. The critical moment for the patient and the family is to understand financial capability, potential and actual possibilities for reimbursement. There is no country that can afford that every patient receives all the best available treatments and even when the best standard of care is recommended in country accepted treatment guidelines, there are waiting lists and this best standard of care is not available to everyone, instantly. However, in developing countries pool of patients that cannot afford expensive treatments and do not have other options, is larger. When designing clinical protocol, Helsinki Declaration (WMA 2013) principles must be followed in the best way possible, in order not to offend human rights of patients with limited treatment options. Prerequisite is that clinical trial is designed including the best standard of care in developed countries, as required by Helsinki Declaration that says: "The benefits, risks, burdens and effectiveness of the new method should be tested against those of the best current prophylactic, diagnostic and therapeutic methods." There is a worldwide accepted understanding that the key requirement of Helsinki Declaration that every patient in clinical trial must receive the best standard of care is not always possible (Lie et al. 2004). However, if physicians who participate and those who develop clinical trials, would not stream to this ideal target and make every effort to achieve it, this will open the door to misinterpretation of this requirement.

Critical aspect that patients usually consider, when deciding to take part in clinical trial, is potential expected benefit for them. Another significant aspect is altruism. As a result, majority of patients taking part in clinical trials, no matter if coming from developed or developing countries, decide to participate in the clinical trial because they would have difficulties to get access to expensive treatments and diagnostics needed for their disease. For them, the clinical trial is an option where they can avoid long waiting lists if they would like to have the treatment reimbursed and diagnostic examinations for free. Even in developed countries there is a segment of patients that would be motivated to participate for the above mentioned reasons. Patients who have good access to various existing treatments, and required treatments are fully reimbursed, do not have motivation to come to numerous visits, to fill in numerous and boring questionnaires and attend long study visits with many blood samplings. Every patient, no matter if comes from developed or developing countries, performs own benefit/risk evaluation taking in consideration benefits and hurdles of clinical trial, mentioned above.

We need to have this in mind when answering the question: "Does it make a difference whether the study was or will be performed in developed countries or in developing countries?", because numerous aspects and differences between developed and developing countries should and can be taken in consideration, but the key seven requirements for ethical research should not be evaluated in different way for developed and developing countries as majority of patients entering clinical trials are coming from population that do not have 
instant access to best treatment options which makes them more vulnerable. No matter from which country they come, independent of country BDP or with percentage of BDP dedicated to the health care, there is a real possibility that they decided to participate because they have limited choices.

\section{SEVEN ETHICAL REQUIREMENTS OF ETHICAL RESEARCH}

There are seven ethical requirements of ethical research per Emanuel (Emanuel et al. 2000):

\section{Value: It must enhance health or derive knowledge that could lead to improvements in health or well being}

If clinical research is designed investigating treatment options which are not considered as the best standard of care in developed countries, results will be non-generalizable, there will be a substantial overlap with already proven results, and research results would most likely not be practically implementable. Resources (e.g. sponsor, government, hospital, etc.) will be diverted from other worthy social pursuits and no new, relevant knowledge will be obtained. It is not ethical to design clinical trial where even negative results do not bring any new knowledge. In the case of unsuccessful clinical trial when efficacy of new drug is not confirmed, at least there is learning that this therapeutic target must be re-evaluated. When drug with known effect is compared to placebo, while best treatment already exists, there is nothing new or unexpected here.

\section{Scientific validity - rigorous methodology}

If scientifically unreliable or invalid results will be obtained by the clinical trial, subjects would be exposed to risk with no purpose. It must be possible to execute the study in any country where the drug is planned to be registered. If it would not be possible to enroll sufficient number of subjects in any other country, this is not the reason to run the study only in few countries, because there are patients who do not have access to the treatment for their disease, so would be grateful to have any treatment. Excluding other countries from research cannot generate valid and relevant scientific knowledge. Results cannot be combined with other similar studies for meta-analysis. Research with no subjects from population where the study medication is planned to be marketed is not relevant for this population, however that does not mean that possibly one day such pharmaceutic cannot find its way to the list of available medicine in that country. If there is a consensus what is the better treatment, there is no null hypothesis and the research is invalid. Without clinical equipoise, research that compares therapy with placebo, when the best treatment is already known, is unlikely to increase knowledge and the benefit/risk ratio will be unfavorable because all subjects in such trial received inferior treatment.
3. Fair subject selection based on scientific objectives, not vulnerable nor privilege, fair distribution of risks and benefits, research not risky, placing some people at risk for harm for benefit of others or no benefit

Population entering the study must be carefully planned and potential risks limited by exclusion and inclusion criteria. Testing of population that is not valid representation of targeted disease population where the drug is planned to be marketed cannot provide relevant knowledge to justify global use. Standard of care recognized in developed countries cannot always be the same in developing countries due to various constraints (e.g. access to multiple sclerosis (MS) treatments in Germany and Poland is different due to percentage of expenditure of BDP that is dedicated to health care, although population is similar and disease characteristics are similar). $13 \%$ of patients suffering from MS were treated in Poland compared to $69 \%$ of patients in Germany (Wilsdon et al. 2014). If clinical trials for new MS treatments will be performed only in Poland, it is difficult to believe that they will provide adequate knowledge about the efficacy of drug in German patients as the severity of the disease may be different. In the same way, knowledge about the efficacy of lapatinib in patients with more progressive course of disease, like in Dr. Guan's study, (Guan et al. 2013) as they were not treated by trastuzumab, is not important and is not relevant for German patients for instance. Due to more progressed disease in Chinese patients, tested drug may not show full efficacy and may never reach the market. In the same way, drug that is efficient in German population with milder disease may not be efficient enough in Chinese patients with more progressed disease. That is the reason why clinical trials are organized to be international and multi-centric. Percentage of patients coming from various races and geographical regions must be carefully followed up in clinical trials, so that efficacy in certain population that is outnumbering the rest of countries, does not drive efficacy results. In both cases described above, research is not ethical if results obtained by this research are not relevant for the overall global knowledge and understanding of the disease.

Development of new drugs only in countries with limited resources and with different standard of care is not justified, as the overall knowledge is not relevant for global population, but also not for the population used in this clinical trial, as drug may never been reimbursed in developing country. There is requirement incorporated in the legislation about clinical trials in some countries like is for instance Ukraine, where is mandatory that patients must continue to be provided by study medication even after the clinical trial ended, until it comes to the market in Ukraine. However, for some drugs, there are no plans to be ever registered in Ukraine and there are various systems in place (e.g. named patient program and compassionate use program) to ensure that those patients will have some benefits from the studied drug. 
In the same way it should be understood that if the new treatment is tested in population with different severity of disease (natural course of disease), then the use in population that is pre-treated and has different severity of the disease should be considered as off label.

Fair subject distribution, must be guided by scientific aims of the research. However, investigator should not be guided by science only, and decision to limit population to subjects with natural course of the disease because they do not have access to standard care, with the aim to understand true efficacy of new treatment, does not justify risks for this population. This does not mean that patient population where the new treatment was tested must directly benefit from the new treatment, but it means that patient population must not be put on unnecessary risk.

\section{Favorable risk/benefit ratio}

Clinical research is organized in a way that by answering to scientific questions obtains new knowledge relevant for clinical practice and relevant for patient's well being. New knowledge cannot always bring benefit to the individual patient but must be relevant for global population. Majority of patients understand this concept and they accept it with sincere altruism that knowledge gained by this research will present benefit for future generations or new patients coming. However, this altruism, patient time, energy, simply speaking "patient blood" must be respected and must not be abused for answering questions that are not relevant, or are already answered by accepted results of well controlled clinical trials.

In order to achieve favorable benefit/risk ratio, there are three conditions that must be fulfilled (Emanuel at al. 2000): potential benefits for individual patient are enhanced, potential risks are minimized and potential benefits for subject and society must outweigh risks.

Even if patients will not benefit from investigated drug or placebo, they will probably benefit from regular study visits, regular laboratory and diagnostic tests and certain amount of placebo coming from physician attention and patient's expectations. However, risk of being not treated properly even during the clinical trial is significantly higher. Potential explanation that those patients would not be treated properly anyway due to limited resources, does not justify their involvement, lost time, energy, blood given for various samples for clinical trial in which they will not have more benefit than placebo effect. Their health condition is already very heavy, severe and serious adverse drug reactions and long study visits are too much burden that can speed up progression of the disease to fatal outcome.

\section{Independent review}

Approval from the local or central country ethics committee is valid and relevant even if it is developing country; however, if the clinical trial was sponsored by organization from developed country, it also expected to obtain relevant ethics committee approval from the rele- vant body in sponsor's country of origin. As per authors (Shapiro \& Meslin 2001): "A review by ethics committees in both the host and sponsoring countries does not guarantee that the trial will be carried out in an ethical manner but does help ensure that both the ethical aspects of the trial and the local context are considered." This is also further explained and emphasized in Nuffield Report (McMillan \& Conlon 2004): "The UK has made moves towards providing this with the work of the ethics committee of the London School of Hygiene and Tropical Medical, The Liverpool School of Tropical Medicine ethical committee and the Oxford Tropical Medical Research Ethics Committee." However, it is not mentioned in Dr Guan's article (Guan et al. 2013) that sponsor company GSK used that expertise in the case of lapatinib clinical trial.

From Landes debate (Landes 2005) we learned that position of the London School of Hygiene and Tropical Medicine is very clear: "Within this broader, and theoretically necessary, understanding of context, it becomes impossible to justify an ethical double standard for research in developing countries."

Clinical Protocols are also usually sent for peer-review to recognized experts in the same field of research, but not affiliated by clinical research. It is possible that in developing countries, expert review is organized in a different way, and that there are other bodies who evaluated the protocol, like is for instance department for clinical pharmacology at the hospital, or members of hospital administration or similar. As we could understand from PPD Whitepaper referring to drug registration for instance in China (PPD Whitepaper 2013), China's regulatory requirements regarding GCP were not completely the same like those incorporated in the USA and EU legislation. We can acknowledge different legal framework and cultural differences, but more effort in providing information how and who assessed the planned clinical study is expected.

As per authors (Wendler at al. 2004): "Specifically, institutional review boards should assume a default of requiring the best methods in all cases and approve research using less than the worldwide best methods only when it satisfies the following 4 conditions:(1) scientific necessity: investigators must use less than the worldwide best methods to answer the scientific question posed by the trial; (2) relevance for the host community: answering the scientific question posed by the trial will help address an important health need of the host community; (3) sufficient host community benefit: the trial will produce a fair level of benefit for the host community; and (4) subject and host community nonmalfeasance: subjects and the host community will not be made prospectively worse off than they would be in the absence of the trial."

\section{Informed consent}

Between January 2006 and December 2009, 444 patients were recruited from seven countries (Russia, Pakistan, Peru, China (mainland and Hong Kong), Thailand, 
Brazil, and Ukraine) (Guan et al. 2013). It is possible that GCP was not fully incorporated in the legal requirements about clinical trials in every of the mentioned countries, and that there are some local differences. The description of Informed Consent process must be provided when publishing study results. In any case, obtaining a signature on paper - in the United States or elsewhere - does not ensure that a participant understands the proposed research. Although signed forms make it easy to audit informed consent - one useful dividend of this process - there are other ways to ensure that it has been obtained. An ethically sound alternative to written consent is oral consent that has been witnessed and verified (Shapiro \& Meslin 2001). When there is no description of the process in the scientific paper describing results of clinical trial, there is an impression that not enough attention was given to that process.

As per authors (Shapiro \& Meslin 2001): "Given these issues, researchers, sponsors, and ethics review committees in developed countries must take great care to ensure that the justification for conducting a trial in a developing country is adequately articulated. This is especially important if the trial is to be conducted in a country or region where the population may be vulnerable to exploitation because of pervasive poverty and disease or lack of understanding of the scientific issues surrounding the health problem and the role of the clinical trial in the search for a solution.

\section{Respect for enrolled subjects}

If the clinical trial is performed only in developing country, valid question is who will benefit from results of that study, as for USA and Western EU patients; standard of care is obviously different. If there is no benefit, enrolled subjects' effort to attend study visits and donate their blood for scientific research is not respected.

\section{ETHICAL JUSTIFICATION OF THE RESEARCH QUESTION}

When designing clinical trials question of using placebo is also very important. New treatments are usually compared to the best standard of care. It must be pointed out that in oncology patient population, placebo arm is not explicitly needed like in psychiatric clinical trials where placebo effect is significant problem. In oncology clinical trials, placebo arm is not considered as ethical, due to severity of the disease and life-threatening condition, so scientific curiosity cannot prevail over the best interest of individual patient. In clinical trials investigating adds on treatments, it is usually add on to the standard of care treatment in comparison to placebo. There is still open question should sponsor provide for free background therapy. In this case it could also be considered as investigational drug, but is usually not expected to be provided for free. There is a point where there is no further ratio- nale for any kind of treatment for metastatic cancer for instance. However, there is no justification (scientific, medical and particularly ethical) that such patients are exposed to increased physical demands connected with study visits until fatal outcome. Clearly, investigators have clinical obligations that go beyond the scientific needs of particular research trials. Investigators cannot justify trials using less than the best methods simply by arguing that in the absence of the trial, subjects would receive nothing (Wendler et al. 2004).

\section{ETHICAL JUSTIFICATION OF THE STUDY DESIGN}

The basic principle involved in the process of reaching a verdict for or against participation in one of the clinical researches includes an evaluation of benifits and risks ratio in regards to executing a clinical trial, with added questioning of moral and medical justification (Vitezović et al. 2014). Study design that was aiming to use as a comparator not widely accepted best standard of care, or placebo, can be partially justified by the fact that those patients will potentially receive access to regular and frequent visits with more frequent laboratory tests and other diagnostic evaluations and tests and better supportive care. Placebo effect coming from patient's expectations and more physician attention cannot be justified in patient population with life threatening diseases. It is difficult to find more benefits for patients that could prevail over risks of being left untreated with metastatic breast cancer if in placebo arm. Even the option of not being treated at all, being left at home, and not being given any medical care, is more acceptable, because at least patients will not be exposed to sometimes difficult transportation to hospital for study visits and various diagnostic tests. It is expected that they are very weak, easily fatigued and that they will not be able to perform even the simple activities of daily living. It is really difficult to justify all this nuisance of study visits without any benefit for an individual patient that is in such a difficult health condition. Only possible benefit that could be procured for patients included in clinical research is providing an improved standard of clinical care compared to the standard service and accommodation offered by a country with bad or lacking resources. Evaluation of objectives and outcomes in medical treatment, of this particlar patient group, shows the necessity of promptness in matters of new pharmaceutical market placement and availability, which will result in multiple benefits for the patient, his doctor and also the drug company. Also, patients with such and other similar ailments, for purposes of clinical trials, should select countries known for fast admission with large enough patient quotas in researches, thus making the pharmaceutic available in as short time as possible (Weigmann 2015, Alemayehu 2018). 


\section{CONCLUSION}

Invalid research is unethical as is waste of resources: of investigator, sponsor and anyone who attends the research (Vanderpool 1996). Without validity the research cannot generate any relevant new knowledge and risks of exposing subjects to potential known and unknown risks cannot be justified (Emanuel et al. 2000). Development of new treatment algorithms is relevant scientific goal and already existing knowledge and guidelines can and must be challenged, since this is the condition for science to be progressive. However, potential risks and benefits of new treatments must be fairly distributed. What is too risky for subjects from developed countries, cannot be acceptable for subjects coming from developing countries. Those who should enjoy benefits should share some risks, and those who are taking risks accepting to be included in testing new treatments must be in position to bear some benefits (Emanuel et al. 2000). Nuffield report suggest that even though a universal standard of care cannot be provided to participants this research should still be conducted because it might help to develop responses to health care needs in developing countries. While we agree with the report's view about this research it is worth considering whether the reasons they give are adequate and justifiable (McMillan \& Conlon 2004). While some studies can be justified as in the case of "the research on HIV in sub-Saharan Africa that can be justified by the extraordinary devastation caused by the epidemic there" (Angell 2000), we have to avoid such justification of clinical trials that are to be performed only in developing countries.

\section{Conflict of interest: None to declare.}

\section{Contribution of individual authors:}

1. Research Project: A. Conception, B. Organization, C. Execution;

2. Statistical Analysis: A. Design, B. Execution, C. Review and Critique;

3. Manuscript Preparation: A. Writing the First Draft, B. Review and Critique.

Marija Bosnjak Pasic: Conception, Organization, Execution, Design, Execution, Writing the First Draft, Review and Critique;

Branka Vidrih: Conception, Organization, Design, Review and Critique

Helena Sarac: Conception, Organization, Design, Review and Critique;

Hanna Pasic: Conception, Design, Review and Critique;

Luka Vujević: Conception, Design, Review and Critique;

Anamarija Soldo Koruga: Conception, Organization, Execution, Execution, Review and Critique;

Fabijan Rajič: Conception, Organization, Design, Review and Critique.

\section{Acknowledgements:}

Financial disclosures for previous 1 month:

Marija Bosnjak Pasic holds a research grant for clinical trial from Sermon, Receptos-Psicro, TEVA PPD and Actelion.

Anamarija Soldo Koruga has Shire, Medis Adria, Merck Serono, Novartis, Actelion, Biogen, Schering financ disclosure,

Branka Vidrih, Helena Sarac, Luka Vujević \& Fabijan Rajič have no financial disclosures.

No writing assitance was utilized in the production of this manuscript.

\section{References}

1. Alemayehu C, Niklas J, Mitchell G: N-of-1 trials in the clinical care of patients in developing countries: a systematic review. Bio Med Central, 2018. Alemayehu C et al.: Trials 19:246. from https://doi.org/10.1186/s13063-0182596-5

2. Angell M: Investigator's responsibilities for human subjects in developing countries. $N$ Engl J Med 2000; 342:967-8

3. Emanuel EJ, Wendler D, Grady C: What makes clinical research ethical? JAMA 2000; 283:2701-11

4. European Medicines Agency (EMA): Europien Union. Agencies and other EU bodies. from https://europa.eu/ european-union/about-eu/agencies/ema_en

5. Guan Z, Xu B, DeSilvio ML, Shen Z, Arpornwirat W, Tong $Z$, et al.: Randomized trial of lapatinib versus placebo added to paclitaxel in the treatment of human epidermal growth factor receptor 2-overexpressing metastatic breast cancer. J Clin Oncol 2013; 31:1947-53

6. Landes M: Can context justify an ethical double standard for clinical research in developing countries? Global Health 2005; 1:11. doi:10.1186/1744-8603-1-11

7. Lie RK, Emanuel E, Grady C, Wendler D: The standard of care debate: the Declaration of Helsinki versus the international consensus opinion. J Med Ethics 2004; 30:190-3

8. McMillan JR, Conlon C: Nuffield Council on Bioethics. The ethics of research related to health care in developing countries. J Med Ethics 2004; 30:204-6

9. PPD Whitepaper: Optimizing Drug Registration in China: Category I Route. Pharmaceutical Product Development, LLC. 2013. Retrieved Nov. \& Dec., 2016, from http://www.ppdi.com/drug-registration-china/China_ CategoryI-Registration-PPD_White\%20Paper.pdf

10. Shapiro HT, Meslin EM: Ethical issues in the design and conduct of clinical trials in developing countries. $N$ Engl $J$ Med 2001; 345:139-42

11. Vanderpool HY, ed.: The ethics of Research Involving Human subjects. Frederick, MD: University Publishing group 1996: $45-58$

12. Verbanac D, Stepanic V: Novi pogled na istraživanje lijekova - nove formulacije i kombinacije. Farmaceutski tehničar: stručno informativni časopis farmaceutskih tehničara Hrvatske, 2013, 7-12. from https://bib.irb.hr/datoteka/618820.Novi_pogled_na_istrazi vanje lijekova_nove formulacije i kombinacije.pdf 
13. Vitezić D, Borovečki A, Vrhovac B, Klinička farmakologija: etika kliničkoga ispitivanja. Medicinska naklada, 2014, str: 87-96.

14. Weigmann $\mathrm{K}$ : The ethics of global clinical trials In developing countries, participation in clinical trials is sometimes the only way to access medical treatment. What should be done to avoid exploitation of disadvantaged populations? EMBO Reports, 2015; 16:566-570. from https://www.ncbi.nlm.nih.gov/pmc/articles/PMC4428044/

15. Wendler D, Emanuel EJ, Lie RK: The standard of care debate: Can research in developing countries be both ethical and responsive to those countries' health needs? Am J Public Health 2004; 94:923-9

16. Wilsdon T, Barron A, Mitchell Heggs A, Ginoza S: Access to medicines for multiple sclerosis: Challenges and opportunities. Charles River Associates, London, February 2014. CRA Project No. D19380. Retrieved Nov. \& Dec., 2016, from http://www.crai.com/sites/default/files/publications/ CRA-Biogen-Access-to-MS-Treatment-Final-Report.pdf

17. World Medical Association: World Medical Association Declaration of Helsinki: ethical principles for medical research involving human subjects. JAMA 2013; 310:2191-4

\section{Correspondence:}

Fabijan Rajič, B.A. in physioth

Croatian Football Federation

Ulica grada Vukovara 267a, 10000 Zagreb, Croatia

E-mail:fabijan.rajic@gmail.com 